\title{
Inaccuracies in Google's Health-Based Knowledge Panels Perpetuate Widespread Misconceptions Involving Infectious Disease Transmission
}

\author{
Andrew D. Haddow ${ }^{1 \star}$ and Sarah C. Clarke ${ }^{2 \star}$ \\ ${ }^{1}$ Virology Division, United States Army Medical Research Institute of Infectious Diseases, Frederick, Maryland; ${ }^{2}$ Darnall Medical Library, \\ Walter Reed National Military Medical Center, Bethesda, Maryland
}

\begin{abstract}
Google health-based Knowledge Panels were designed to provide users with high-quality basic medical information on a specific condition. However, any errors contained within Knowledge Panels could result in the widespread distribution of inaccurate health information. We explored the potential for inaccuracies to exist within Google's health-based Knowledge Panels by focusing on a single well-studied pathogen, Ebola virus (EBOV). We then evaluated the accuracy of those transmission modes listed within the Google Ebola Knowledge Panel and investigated the pervasiveness of any misconceptions associated with inaccurate transmission modes among persons living in Africa. We found that the Google Ebola Knowledge Panel inaccurately listed insect bites or stings as modes of EBOV transmission. Our scoping review found 27 articles and reports that revealed that 9 of 11 countries where misconceptions regarding insect transmission of EBOV have been reported are locations of current (i.e., Democratic Republic of Congo and Guinea) or previous EBOV outbreaks. We found reports that up to $26.6 \%$ (155/582) of study respondents in Democratic Republic of Congo believed mosquito bite avoidance would prevent EBOV; in other locations of previous large-scale EBOV outbreaks (e.g., Guinea), up to 61.0\% (304/498) of respondents believed insects were involved in EBOV transmission. Our findings highlight the potential for errors to exist within the health information contained in Google's health-based Knowledge Panels. Such errors could perpetuate misconceptions or misinformation, leading to mistrust of health workers and aid agencies and in turn undermining public health education or outbreak response efforts.
\end{abstract}

\section{INTRODUCTION}

Due to the widespread use of Google for health-related searches, Google unveiled their health information-based "Knowledge Graphs" in 2015. ${ }^{1}$ Although general topic Knowledge Graphs, also known as Knowledge Panels, have existed for years, ${ }^{2}$ health-based Knowledge Panels were designed to provide users with basic medical information on a specific condition. These Knowledge Panels feature prominently on search engine results pages and appear as the first entry when using mobile devices for Web searches, substantially increasing the likelihood that users will use them for information when scanning their search results. ${ }^{3}$

To generate a health-based Knowledge Panel, Google selects and analyzes information from websites determined to be of high quality; then, teams of doctors review and edit the information for accuracy. ${ }^{4}$ Following a Google search for a specific medical condition, the resulting Knowledge Panel (when available) is conspicuously displayed at the top of the search results. This Knowledge Panel provides a summary or overview of the medical condition and may include transmission modes, symptoms, treatments, and whether the condition requires diagnosis and treatment by a medical professional (Figure 1).

In 2018, a study focusing on Ebola virus (EBOV) knowledge, attitudes, and practices in the Democratic Republic of Congo (DR Congo) reported that $26.6 \%$ of respondents believed the virus could be transmitted via mosquito bite (Michael Hawkes, personal communication). ${ }^{5}$ That report, and the substantial increase in the peer-reviewed and grey literature covering

\footnotetext{
*Address correspondence to Andrew D. Haddow, Virology Division, United States Army Medical Research Institute of Infectious Diseases, 1425 Porter Street, Frederick, MD 21702, E-mail: andrew.d.haddow. ctr@mail.mil or Sarah C. Clarke, Darnall Medical Library, Walter Reed National Military Medical Center, 8901 Wisconsin Ave., Bethesda, MD 20889, E-mail: sarah.c.clarke.civ@mail.mil.
}

EBOV transmission in the years following the 2013-2016 West African Ebola virus disease (EVD) outbreak, indicated the potential for inaccuracies relating to EBOV transmission modes to exist on the Internet. Such inaccuracies, combined with poor eLiteracy skills, could lead to an overestimation of risk; create fear, mistrust, and rumors; perpetuate misinformation; and ultimately require labor-intensive public health education efforts to dispel misconceptions.

Herein, we evaluated the accuracy of information pertaining to the EBOV transmission modes listed in the associated Google Knowledge Panel. We then carried out a scoping review of the knowledge and attitudes of persons living in Africa regarding those inaccurate transmission modes we found were listed in the Google Ebola Knowledge Panel.

\section{METHODS}

Assessment of accuracy. We carried out a search in Google on March 8, 2021 using the terms: "Ebola," "Ebola virus," "Ebola hemorrhagic fever," and "Ebola virus disease." All search terms resulted in the same Knowledge Panel (Figure 1). Transmission modes listed in the Google Ebola Knowledge Panel were then cross-checked against those transmission modes listed on the $\mathrm{WHO}^{6}$ and the $\mathrm{CDC}^{7} \mathrm{EBOV}$ webpages for accuracy (Supplemental Figure 1).

Scoping review search strategies and selection criteria. After we determined inaccuracies existed within the Google Ebola Knowledge Panel relating to EBOV transmission by insects or stings (Supplemental Figure 2), we carried out a scoping review to determine the knowledge and attitudes of persons living in Africa as related to these transmission modes.

Literature searches were performed on March 8, 2021 using the platforms PubMed and Web of Science, in addition to the database African Index Medicus. Keywords were identified and searched by the authors to identify literature addressing EBOV knowledge and attitudes in African countries as related 


\section{Ebola \\ Also called: ebola hemorrhagic fever \\ OVERVIEW SYMPTOMS TREATMENTS SPECIALISTS}

A virus that causes severe bleeding, organ failure, and can lead to death.

Humans may spread the virus to other humans through contact with bodily fluids such as blood.

Initial symptoms include fever, headache, muscle pain, and chills. Later, a person may experience internal bleeding resulting in vomiting or coughing blood.

Treatment is supportive hospital care.

\section{Extremely rare \\ Fewer than 1,000 US cases per year \\ Treatable by a medical professional \\ Spreads by animals or insects \\ Requires a medical diagnosis \\ Lab tests or imaging always required \\ Critical: needs emergency care}

\section{HOW IT SPREADS}

By blood products (unclean needles or unscreened blood).

By animal or insect bites or stings.

By touching a contaminated surface (blanket or doorknob)

By saliva (kissing or shared drinks).

For informational purposes only. Consult your local medical authority for advice.

Sources: Mayo Clinic and others. Learn more

FIGURE 1. Google Knowledge Panel, sometimes referred to as a Knowledge Graph or Infobox, for searches using the terms: "Ebola," "Ebola virus," "Ebola hemorrhagic fever," and "Ebola virus disease." Searches were carried out on January 12, 2021. All three searches resulted in the same Knowledge Panel. This figure appears in color at www.ajtmh.org.

to the transmission of EBOV by either insects or stings. The following keywords were used in conjunction with Boolean operators to form search statements for PubMed and Web of Science: arthropod, attitude, bite, Culicidae, Ebola, flies, fly, housefly, insect, knowledge, mosquito, Muscidae, and sting. The African Index Medicus was searched more broadly. Search strategies can be found in the Supplemental Methods. Search statements were designed to retrieve literature mentioning Ebola and virus transmission via insects or stings or articles focused on knowledge and attitudes surrounding EBOV or EVD. Database searches were supplemented by citation pearl searching or by screening reference lists of inclusion literature and grey literature to produce additional publications for review. Bibliographic records, including abstracts, were exported from the search results into EndNote X8 (Clarivate Analytics, Philadelphia, PA). In EndNote X8, the Find Duplication function was used, followed by further manual deduplication.

Abstracts available in English were screened for information regarding African knowledge, attitudes, and/or misconceptions related to EBOV transmission via insects or stings. Retracted articles were excluded. Full-text articles were then read to determine eligibility, including all articles that specifically stated "knowledge" and "attitudes" in the title due to the potential of in-scope criteria in figures. Literature was excluded if it did not report knowledge, attitudes, and/or misconceptions as related to EBOV transmission by insects or stings. Full-text literature was also excluded if the full text of the article or report was in a non-English language. However, non-English articles with a translated abstract were included if they met our selection criteria.

\section{RESULTS}

Transmission mode inaccuracies. We determined that the Google Ebola Knowledge Panel (Figure 1) incorrectly listed "[Ebola virus] spreads by ... insects" and "How it [Ebola virus] spreads: By . . . insect bites or stings" as modes of virus transmission.

Attitudes and misconceptions. A total of 27 articles or reports met the inclusion criteria for our scoping review (Supplemental Figure 3). We found widespread misconceptions existed in those regions with previous EVD outbreaks as well as in regions in proximity to previous outbreaks (e.g., EBOV is transmitted by insects) (Table 1). Importantly, 9 of the 11 countries where misconceptions regarding insect transmission of EBOV were reported are locations of current (i.e., DR Congo and Guinea) or previous EVD outbreaks. Alarmingly, two recent reports from DR Congo indicated that $24.8 \%$ and $26.6 \%$ of respondents felt that EBOV transmission to people could be prevented by avoiding mosquito bites. In other locations of previous large-scale EVD outbreaks, up to $61.0 \%$ of interviewees/respondents in some surveys felt insects were involved in the transmission of EBOV. One study carried out in the DR Congo and another study carried out in Ghana that combined multiple variables, including knowledge, attitudes, or misconceptions involving EBOV insect transmission, among others, were not included in Table 1.8,9 Our scoping review did not find any reports indicating persons believed EBOV transmission occurred by "stings."

\section{DISCUSSION}

We determined that inaccuracies exist within the Google Ebola Knowledge Panel relating to virus transmission by insects or stings (Figure 1). To date, peer-reviewed research does not support the role of insects in the transmission of EBOV to humans. ${ }^{10-13}$ Additionally, our searches did not find any peer-reviewed evidence suggesting EBOV is transmitted to humans by "stings." The Knowledge Panel indicates the information contained was sourced from the "Mayo Clinic and others." However, the Mayo Clinic EBOV linked webpage states: "There's no evidence that Ebola virus or Marburg virus can be spread via insect bites."14 It therefore remains unclear how the incorrect EBOV transmission modes ended up in the Knowledge Panel as the information contained within undergoes expert review prior to publication ${ }^{4}$ or how long this information has been publicly available. Google's Knowledge Panels allow users to submit feedback, which the authors submitted on May 31, 2019 to alert Google to the inaccurate 
TABLE 1

Studies carried out in Africa reporting misconceptions associated with Ebola virus transmission via insects

\begin{tabular}{|c|c|}
\hline Location & Population interviewed/surveyed \\
\hline $\begin{array}{l}\text { Democratic } \\
\text { Republic of } \\
\text { Congot }\end{array}$ & $\begin{array}{l}\text { Public ( } \geq 14 \text { yr old) } \\
\text { Public ( } \geq 18 \text { yr old) }\end{array}$ \\
\hline Ethiopia & $\begin{array}{l}\text { Health care providers working in a } \\
\text { hospital setting ( } \geq 20 \mathrm{yr} \text { old) }\end{array}$ \\
\hline Gabon $\ddagger$ & Public and some health care providers \\
\hline Ghana & $\begin{array}{l}\text { Public and some health care providers } \\
\text { ( } \geq 20 \mathrm{yr} \text { old })\end{array}$ \\
\hline Guinea†ף & $\begin{array}{l}\text { Public (preference given to head-of- } \\
\text { household, } \geq 18 \mathrm{yr} \text { old) }\end{array}$ \\
\hline & $\begin{array}{l}\text { Public (head-of-household and an adult } \\
\text { woman or young person } 15-24 \text { yr old) } \\
\text { Public (head-of-household and an adult } \\
\text { woman } \geq 25 \text { yr old or young person } \\
15-24 \text { yr old) } \\
\text { Public (head-of-household and an adult } \\
\text { woman } \geq 25 \text { yr old or young person } \\
15-24 \text { yr old) }\end{array}$ \\
\hline Liberia & Public and some health care providers \\
\hline
\end{tabular}

Head-of-household or designated individuals when the head-ofhousehold was unavailable $(\geq 18 \mathrm{yr}$ old $)$ EVD actively monitored or quarantined households (head-of-household)

Nigeriał Public and healthcare providers $(\geq 18 \mathrm{yr}$ old)

Clinicians

Hospital clinic ( $\geq 20 \mathrm{yr}$ old)

Public ( $\geq 18$ yr old)

Medical and nursing students

Senegalł $\quad$ Vaccine trial participants $(\geq 18 \mathrm{yr}$ old $)$

Sierra Leone Public (head-of-household and an adult woman $\geq 25 \mathrm{yr}$ old or a young person 15-24 yr old)

Public (head-of-household and an adult woman $\geq 25$ yr old or a young person 15-24 yr old)

Healthcare providers

Health care providers $(\geq 20 \mathrm{yr}$ old $)$

Public ( $\geq 18$ yr old)

Ugandał

West Africa and South Africa\#,**
Public areas of previous EVD/MVD outbreaks ( $\geq 18$ yr old)

Academicians (scientific fields) with degrees or those without degrees (18-45 yr old)

Year of interview/survey
August 2018
August 2018
August-October
2015
$\leq 2001^{\|}$
February-March
2015
December $2014-$
January 2015
August 2015
August 2015
August 2015
August 2014

"Can protect self from Ebola by avoiding mosquito bites [No. $155 / 582(26.6 \%)] \S^{\prime \prime}$

"Ebola can be prevented by avoiding mosquito bites [No. 71/286 $(24.8 \%)]^{\prime 25}$

"But many believed that EVD can be transmitted through ... or [other insects] mosquitoes (24.3\%) [No. 52/211]"26

"... others responded the mosquitoes ... were the cause [of the EBOV outbreak]" 27\|

"there was a firm belief that Ebola ... could also be transmitted through air, mosquito bites and houseflies" 28

"... major misconceptions that need to be rejected include perceptions that EVD is transmitted by ... mosquito [No. 94/ $345(27.3 \%)]^{\prime 29}$

“... in Forécariah 61\% [No. 304/498] of respondents believed Ebola can be transmitted through mosquito bites ..."30

“... [No. 2,795/5,705] 49\% believed they could protect themselves from Ebola by avoiding mosquito bites" ${ }^{31}$

"Reported mosquito bites as [sic] cause of Ebola (57\%) [No. 3268/5733]"16

" ... at the onset of the emergency phase of the epidemic [EBOV], the Government of Liberia launched a public health campaign that enjoyed [sic] people to ... protect themselves from insect bites (a carryover from antimalarial campaigns) ..."19 "As a result, local populations initially believed that a number of non-Ebola sources of infection, like insects ... were primary sources for Ebola."19

December 2014

“...33\% [No. 387/1,190] of respondents believed Ebola could be spread by mosquito bites ...",20

April 2015

2014

August-September 2014

October-December 2014

2014-2015

August-September 2015

August-November 2015

August 2014

August 2014

"However, interviewees sometimes incorrectly identified mosquito bites [No. 67/115 (58.3\%)], as a mode of EBOV transmission..." 32

“...26\% [of respondents] think it [EBOV] could be spread by mosquito bite..."33

"Transmission through mosquito bite [No. 80/398 (20.1\%)]

“. . . believed. . [No. 32/400] (8.0\%) from mosquito bites . ..”35

"How is Ebola spread? . . . Through insect bites [No. 62/438 $(14.2 \%)]^{36}$

"[Routes of transmission of EVD are] . . by mosquito bites [No. $57 / 423(13.5 \%))^{337}$

"The others [sic] transmission mode identified by interviewees: mosquito bites [No. 109/249 (43.8\%)]..."38

"Ebola can be transmitted by mosquito bites (30\%) [No. 424/ 1413]"39

“... [EBOV is] transmitted through: mosquito bites (29.6\%) [No. 403/1360]"40

February 2017

Prior to training: "Ebola is transmitted through mosquito bites [No. 9/99 (9.1\%)]"21

Post training: "Ebola is transmitted through mosquito bites [No. $4 / 93(4.3 \%)]^{21}$

“. . [ [No. 52/258 (20.2\%)] identified insects as a mode of EVD transmission" ${ }^{\prime 1}$

November-

December 2014

May 2015

"[Respondents reporting transmission was] vector born [sic] $12.9 \%$ [No. 85/655]...,"42

January-February

2015

2014-2015
“...11.3\% [No. 60/530] thought that EVD/MVD could be transmitted through biting mosquitoes ..."43

“...23\% [No. 23/100] believed that insects and mosquitoes can help [sic] in transmitting Ebola Virus Disease..."44

EBOV = Ebola virus; $\mathrm{EVD}$ = Ebola virus disease; $\mathrm{MVD}=$ Marburg virus disease

${ }^{*}$ Frequency data are reported as number (\%) whenever possible. Some articles reported the percentage without the denominator from which the percentage was derived, the percentage including the denominator from which the percentage was derived, the percentage without the numerator and denominator from which the percentage was derived, the percentage including the numerator and denominator from which the percentage was derived, or the numerator or denominator in the text from which the percentage was derived was unclear.

$\dagger$ Current or recent Ebola virus transmission.

$\ddagger$ Countries reporting previous Ebola virus outbreaks.

§Michael Hawkes, personal communication, September 4, 2019.

"This publication was written in Japanese; therefore, we used the available English translation of the abstract for the associated information. The year the interviews were conducted was not available in the abstract.

I Countries with widespread Ebola virus transmission or cases during the 2013-2016 outbreak in West Africa.

\# Countries reporting limited Ebola virus cases ( $\leq 2$ cases).

${ }_{\text {\#* Reported response results from West African and South African participants were not separated by region or country but were combined. }}^{\text {\# }}$ 
content. Surprisingly, the Knowledge Panel also lacked information on other important transmission modes (e.g., sexual transmission).

We found numerous articles reporting widespread misconceptions among populations in countries with previous EVD outbreaks that the virus was transmitted by insects. Notably, of the 11 countries in Africa that reported EVD outbreaks since 1976, including those involving the Sudan, Taï Forest, or Bundibugyo viruses, nine countries had populations that believed EBOV was transmitted by insects at the time of the questionnaires/surveys. These findings underscore that reliable information from publicly accessible sources is critical to preventing misconceptions and rumors in regions with active EBOV transmission. Inaccuracies regarding health information might be exacerbated in regions experiencing civil unrest, such as the DR Congo, where there is already substantial mistrust and active public health disinformation campaigns being carried out by violent non-state actors. ${ }^{15-18}$

Inaccuracies in the Google Ebola Knowledge Panel could also have the unintended consequence of perpetuating inaccurate public health information leading to additional confusion regarding EBOV transmission modes. In 2015, during the EVD outbreak, the Liberian government launched a "onestop shop" campaign to provide preventative disease information. ${ }^{19}$ The circulating messages encouraged several tips for preventing EVD, including protection against insect bites (previously part of antimalarial efforts). Although this message would have had the benefit of potentially reducing malaria and arbovirus transmission, it caused confusion among local populations because it indicated that insects (e.g., mosquitoes) were involved in the transmission of EBOV. ${ }^{19}$ One report even stated, ". . . common misconceptions, especially about mosquitoes need to be addressed."20 Of note, training of health care workers focused on EBOV transmission modes has been shown to have a positive effect in reducing the perception that mosquitoes are involved in virus transmission. ${ }^{21}$

There are limitations to our study. Although $41.0 \%$ of online persons living in sub-Saharan Africa use the internet to get health information ${ }^{22}$ and Google accounts for $97.3 \%$ of the African search engine market share (as of February 2021), ${ }^{23}$ it is possible those individuals seeking information on EBOV or EVD use search engines other than Google or use sources other than the internet for health information (e.g., radio, television, and/or community meetings). Even so, Google Trends search interest data from Google "Ebola" searches made within DR Congo from 2017 to 2020 (during EVD outbreaks) showed above-average interest in this topic, ${ }^{24}$ with spikes of tremendous interest (when compared with all other Google searches made within DR Congo) coinciding with public health announcements regarding new outbreaks or case reports. PubMed and Web of Science do not allow for the full text of an article to be searched; therefore, literature containing keywords in the body of the text may have been missed. Additionally, African health and medical journals are often unindexed in international bibliographic databases, so locating knowledge, attitude, and practice research from Africa cannot be accomplished exclusively by searching PubMed and Web of Science. To mitigate this, we also searched African Index Medicus. Finally, some of the studies included in our table may have inadvertently used the same data set, and the survey response rates in some reports were unclear.
Although Google is a cost-efficient and timely source for health information easily accessible to anyone with internet access, users must use eLiteracy skills to understand and appraise online information when addressing health concerns because errors in even "trusted sources" are likely commonplace. For example, we carried out a quick preliminary search of several other Google health-based Knowledge Panels, which we found also contained inaccuracies associated with pathogen transmission modes. These inaccurate Knowledge Panels include rabies and incorrectly state that rabies "...spreads by insect bites or stings," and that transmission of several vector-borne pathogens (e.g., Zika, dengue, chikungunya, yellow fever, malaria, and Lyme disease) may also occur through "stings," which is a misnomer.

Our results highlight the need for health-related "Knowledge Panels, Knowledge Graphs, or Infoboxes" provided by various Web search engines to provide accurate information and use rigorous fact checking prior to publishing. Errors found within Knowledge Panels could perpetuate misconceptions, misinformation, and/or rumors involving infectious diseases and could potentially lead to further mistrust of health workers and aid agencies, severely undermining public health education and outbreak response efforts.

Received February 22, 2021. Accepted for publication March 12, 2021.

Published online April 26, 2021.

Note: Supplemental material appear at www.ajtmh.org.

Disclaimer: The opinions, interpretations, conclusions, and recommendations herein are those of the authors and are not necessarily endorsed by the U.S. Army, U.S. Department of Defense, or U.S. Government.

Authors' addresses: Andrew D. Haddow, Virology Division, United States Army Medical Research Institute of Infectious Diseases, Frederick, MD, E-mail: andrew.d.haddow.ctr@mail.mil. Sarah C. Clarke, Darnall Medical Library, Walter Reed National Military Medical Center, Bethesda, MD, E-mail: sarah.c.clarke.civ@mail.mil.

\section{REFERENCES}

1. Ramaswami P, 2015. A Remedy for Your Health-Related Questions: Health Info in the Knowledge Graph. Available at: https:// www.blog.google/products/search/health-info-knowledge-graph/ . Accessed January 13, 2021.

2. Singhal A, 2012. Introducing the Knowledge Graph: Things, Not Strings. Available at: https://www.blog.google/products/search/ introducing-knowledge-graph-things-not/. Accessed January 13, 2021.

3. Moran K, Goray C, 2019. Complex Search-Results Pages Change Search Behavior: The Pinball Pattern. Available at: https:// www.nngroup.com/articles/pinball-pattern-search-behavior/. Accessed January 13, 2021.

4. Google, 2021. Search for Medical Information on Google. Available at: https://support.google.com/websearch/answer/2364942? $\mathrm{p}=$ medical_conditions\&hl=en\&visit_id=636950356120451051$3942427271 \&$ rd=1. Accessed January 13, 2021.

5. Claude KM, Underschultz J, Hawkes MT, 2018. Ebola virus epidemic in war-torn eastern DR Congo. Lancet 392: 1399-1401.

6. WHO, 2021. Ebola Virus Disease. Available at: https://www. who.int/news-room/fact-sheets/detail/ebola-virus-disease. Accessed January 13, 2021.

7. CDC, 2021. Ebola (Ebola Virus Disease) Transmission. Available at: https://www.cdc.gov/vhf/ebola/transmission/index.html. Accessed January 13, 2021. 
8. Tenkorang EY, 2018. Effect of knowledge and perceptions of risks on Ebola-preventive behaviours in Ghana. Int Health 10: 202-210.

9. Wirsiy FS et al., 2018. Assessment of the knowledge, attitude and practice of pygmies towards the transmission of Ebola viral disease in the Congo basin: a descriptive and analytic crosssectional study. J Environ Sci Health 2: 196-209.

10. Haddow $A D$ et al., 2017. Low potential for mechanical transmission of Ebola virus via house flies (Musca domestica). Parasit Vectors 10: 218

11. Kuhn J, Calisher $\mathrm{CH}, 2008$. Filoviruses: $A$ Compendium of 40 Years of Epidemiological, Clinical, and Laboratory Studies. New York, NY: Springer.

12. Turell MJ, Bressler DS, Rossi CA, 1996. Short report: lack of virus replication in arthropods after intrathoracic inoculation of Ebola Reston virus. Am J Trop Med Hyg 55: 89-90.

13. Swanepoel $R$ et al., 1996. Experimental inoculation of plants and animals with Ebola virus. Emerg Infect Dis 2: 321-325.

14. Mayo Clinic, 2021. Ebola Virus and Marburg Virus. Available at: https://www.mayoclinic.org/diseases-conditions/ebola-virus/ symptoms-causes/syc-20356258?utm_source=Google\&utm medium=abstract\&utm_content=Ebola\&utm_campaign= Knowledge-panel. Accessed January 13, 2021.

15. Trapido J, 2019. Ebola: public trust, intermediaries, and rumour in the DR Congo. Lancet Infect Dis 19: 457-458.

16. Irwin $\mathrm{KL}$ et al., 2017. Attitudes about vaccines to prevent Ebola virus disease in Guinea at the end of a large Ebola epidemic: results of a national household survey. Vaccine 35 (49 Pt B): 6915-6923.

17. Masumbuko Claude K, Underschultz J, Hawkes MT, 2019. Social resistance drives persistent transmission of Ebola virus disease in Eastern Democratic Republic of Congo: a mixed-methods study. PLoS One 14: e0223104.

18. Vinck P, Pham PN, Bindu KK, Bedford J, Nilles EJ, 2019. Institutional trust and misinformation in the response to the 2018-19 Ebola outbreak in North Kivu, DR Congo: a population-based survey. Lancet Infect Dis 19: 529-536.

19. Abramowitz S, McKune SL, Fallah M, Monger J, Tehoungue K, Omidian PA, 2017. The opposite of denial: social learning at the onset of the Ebola emergency in Liberia. $J$ Health Comm 22 (Suppl 1): 59-65.

20. Ministry of Health Liberia, 2015. National Knowledge, Attitudes and Practices (KAP) Study on Ebola Virus Disease in Liberia. Monrovia, Liberia: Ministry of Health Liberia.

21. Reidy $P$ et al., 2017. Knowledge, attitudes and practice for Ebola virus disease in the national rapidly deployable isolation and treatment facility in Sierra Leone. J Infect 74: 618-620.

22. Silver L, Johnson C, 2018. Internet Connectivity Seen as Having Positive Impact on Life in Sub-Saharan Africa. Washington, DC: Pew Research Center.

23. StatCounter, 2021. GlobalStats. Available at: http://gs.statcounter. com/search-engine-market-share/all/africa. Accessed February 9, 2021.

24. Google, 2021. Google Trends. Available at: https://trends.google. $\mathrm{com} /$ trends/explore?date=today\%205-y\&geo=CD\&q=ebola. Accessed February 9, 2021.

25. Kasereka MC, Hawkes MT, 2019. The cat that kills people: community beliefs about Ebola origins and implications for disease control in Eastern Democratic Republic of the Congo. Pathog Glob Health 113: 149-157.

26. Abebe TB et al., 2016. Healthcare professionals' awareness, knowledge, attitudes, perceptions and beliefs about Ebola at Gondar University Hospital, Northwest Ethiopia: a crosssectional study. J Public Health Africa 7: 570.

27. Kunii O, Kita E, Shibuya K, 2001. Epidemics and related cultural factors for Ebola hemorrhagic fever in Gabon. Nippon Koshu Eisei Zasshi 48: 853-859.
28. Adongo PB, Tabong PT, Asampong E, Ansong J, Robalo M, Adanu RM, 2016. Beyond knowledge and awareness: addressing misconceptions in Ghana's preparation towards an outbreak of Ebola virus disease. PLoS One 11: e0149627.

29. Buli BG et al., 2015. Misconceptions about Ebola seriously affect the prevention efforts: KAP related to Ebola prevention and treatment in Kouroussa Prefecture, Guinea. Pan Afr Med J 22 (Suppl 1): 11.

30. Jalloh MF et al., 2017.Assessments of Ebola knowledge, attitudes and practices in Forécariah, Guinea and Kambia, Sierra Leone, July-August 2015. Philos Trans of R Soc B 372: 20160304.

31. Jalloh MF et al., 2017. Knowledge, attitudes, and practices related to Ebola virus disease at the end of a national epidemic Guinea, August 2015. MMWR 66: 1109-1115.

32. Wilken JA et al., 2017. Knowledge, attitudes, and practices among members of households actively monitored or quarantined to prevent transmission of Ebola virus disease - Margibi County, Liberia: February-March 2015. Prehosp Disaster Med 32: 673-678.

33. Center for Public Policy Alternatives, 2014. Study on the Ebola Virus Disease (EVD) Knowledge, Attitudes and Practices of Nigerians in Lagos State. Lagos, Nigeria: Center for Public Policy Alternatives.

34. Ajayi NA, Ojide CK, Ajayi IA, Ukwaja KN, 2019. Evaluation of clinicians' knowledge of and attitudes to Ebola virus disease in Ebonyi State, Nigeria. J Virus Erad 5: 145-151.

35. Shittu R, Sanni M, Odeigah L, Akanbi I, Aderibigbe S, 2015. Awareness, knowledge and misconceptions about Ebola virus disease (EVD) in a family practice setting in Nigeria, West Africa. $J$ Antivir Antiretrovir 7: 10-14.

36. Kaoje AU, Yahaya M, Sabir AA, Raji MO, Abdulmumin S, Mohammed AU, 2016. Awareness, knowledge, and misconceptions of Ebola virus disease among residents of a rural community in Sokoto, Northwest Nigeria. Ann Trop Med PH 9: 105.

37. Balami LG, Ismail S, Saliluddin SM, Garba SH, 2016. Predictors of knowledge regarding Ebola virus disease among medical and nursing students in a Nigerian teaching hospital. Int $\mathrm{J}$ Community Med Public Health 3: 3123-3129.

38. Lakhe NA et al., 2018. Knowledge, attitudes and practices towards Ebola Virus Disease among participants in an Ebola vaccine trial in Dakar, Senegal. J Trop Dis 6: 1000276.

39. Jalloh MF et al., 2017. National survey of Ebola-related knowledge, attitudes and practices before the outbreak peak in Sierra Leone: August 2014. BMJ Glob Health 2: e000285.

40. Catholic Relief Services, 2014. Study on Public Knowledge, Attitudes, and Practices Relating to Ebola Virus Disease (EVD) Prevention and Medical Care in Sierra Leone. Baltimore, MD: Catholic Relief Services.

41. Alfaki MM, Salih AM, Elhuda DA, Egail MS, 2016. Knowledge, attitude and practice of health care providers toward Ebola virus disease in hotspots in Khartoum and White Nile states, Sudan, 2014. Am J Infect Control 44: 20-23.

42. Mohamed MMG et al., 2017. Ebola hemorrhagic fever under scope, view of knowledge, attitude and practice from rural Sudan in 2015. J Infect Public Health 10: 287-294.

43. Nyakarahuka L et al., 2017. Knowledge and attitude towards Ebola and Marburg virus diseases in Uganda using quantitative and participatory epidemiology techniques. PLoS Negl Trop Dis 11: e0005907.

44. Oluwagbemi O, Oluwagbemi F, Abimbola O, 2016. Ebinformatics: Ebola fuzzy informatics systems on the diagnosis, prediction and recommendation of appropriate treatments for Ebola virus disease (EVD). Inform Med Unlocked 2: 12-37. 